\title{
HISTORIA PATRIA Y CURRÍCULO OFICIAL. DOS LUCHAS FRATRICIDAS DE COLOMBIA ANALIZADAS A PARTIR DE LA HISTORIA A ENSEÑAR, 1903-1984*
}

\author{
NATIONAL HISTORY AND OFFICIAL CURRICULUM. \\ TWO FRATRICIDAL STRUGGLES OF COLOMBIA ANALYZED \\ FROM THE HISTORY TO TEACH, 1903-1984
}

Nilson Javier Ibagón Martín ${ }^{* *}$

\begin{abstract}
A partir del análisis de contenido de disposiciones curriculares oficiales y manuales escolares que regularon la enseñanza de la historia de Colombia de 1903 a 1984, se identifican los mecanismos con los que discursivamente el Estado vía institución escolar, buscó dar cuenta de dos procesos de luchas fratricidas que marcaron la historia del país a lo largo del siglo XX: "La Guerra de los Mil Días" y el período de "La Violencia". En este sentido, a partir de la centralidad que tuvo la difusión de la Historia Patria, el artículo estudia la configuración de una memoria oficial que, amparada en la defensa de las ideas de "reconciliación" y "unidad" nacional, se negó a establecer una comprensión crítica de la conflictividad sociopolítica del país, promoviendo con ello el olvido y tergiversación de procesos y acontecimientos asociados a diferentes hechos de violencia que definieron la historia del siglo XX de Colombia.
\end{abstract}

Palabras claves: Currículo Oficial, Manuales escolares, Violencia, Enseñanza de la Historia, Historia Patria, Colombia

From the content analysis of the official curricular dispositions and the textbooks that regulated the teaching of Colombian history from 1903 to 1984, we identify the way through which the State discursively through schooling education, sought to account for two processes of fratricidal struggles that marked the country`s history throughout in the twentieth century; "The War of the Thousand Days" and the period of "La Violencia". In this sense, from the centrality that had the diffusion of the National History, the article studies the configuration of an official memory which that based on the defense of the ideas of national "reconciliation" and "unity", refused to establish a critical understanding of the socio-political conflict of the country, thus promoting the oblivion and distortion of processes and events associated with different acts of violence that defined the history of the twentieth century in Colombia.

Key words: Official Curriculum, Textbooks, Violence, Teaching History, National History, Colombia.

\section{Introducción}

La historia del siglo XX colombiano ha estado marcada por diferentes procesos de violencia fratricida que han puesto en entredicho el proyecto de nación. Violencia que hoy sigue afectando a diferentes sectores sociales y grupos poblacionales. Estas realidades hacen compleja la construcción y difusión de una historia, que, al estar definida en buena parte por la confrontación y el conflicto armado, ha puesto en cuestionamiento la idea de "unidad" desde donde las elites buscan estructurar metarrelatos que tienen como intención dar sustento y validez al Estado-nacional.
El presente escrito propone desde el reconocimiento de esta dificultad y la complejidad ética, emocional e intelectual que supone el abordaje de ciertos episodios incómodos del pasado que caracterizan la historia de Colombia (Ibagón, 2019; Ibagón y Chisnes, 2019; Padilla y Bermúdez, 2016), entender las formas en las que discursivamente el Estado colombiano a lo largo del siglo XX, afrontó el problema que suscitaba dar cuenta de una historia marcada por el ejercicio de una violencia sistemática y la confrontación armada entre connacionales. Así pues, el artículo se estructura desde un doble propósito, por un lado propone un análisis crítico de dos

\footnotetext{
* Resultado del proyecto "Representaciones Sociales del período de la Violencia en textos escolares colombianos, 1989-2016", trabajo adscrito a la línea de investigación "Métodos, enseñanza y divulgación de la Historia" del Grupo de Investigación IHEPE del Departamento de Historia de la Universidad del Valle.

** Universidad del Valle. Cali, Colombia. Correo electrónico: nilson.ibagon@ correounivalle.edu.co
} 
momentos emblemáticos de la historia conflictiva de Colombia: la Guerra de los Mil Días y el período de la violencia ${ }^{1}$-eventos cuyas causas y consecuencias se convirtieron en realidades complicadas de procesar por parte de un sistema educativo y un saber escolar -la historia-, que tenían como objetivo prioritario fortalecer la identidad nacional-; y por otro, establece elementos de análisis y discusión de las formas y sentidos desde los que se abordaron estos procesos históricos en el currículo oficial colombiano.

De ahí que se haya definido como eje central de análisis, el estudio de los discursos educativos oficiales que orientaron la enseñanza de la historia en el país en un período que va de 1903 a 1984. Las coyunturas que definen el marco temporal de indagación se sustentan en referentes curriculares asociados respectivamente a la ley 39 de 1903 Ley orgánica de instrucción- y el Decreto 1002 de 1984. La primera instituye la noción de Historia Patria en el sistema escolar y, la segunda, mediante el principio de integración curricular de las Ciencias Sociales escolares, debilita dicha noción. Además, este marco temporal se sustenta a partir de dos principios analíticos. Por un lado, es un período en el que escolarmente se configura la primacía de la llamada Historia Patria como único referente para entender la historia del país ${ }^{2}$. Por otro lado, es un período en el que en el escenario práctico se dan diferentes ciclos de violencia, los que suscitan inmediatamente una posición oficial educativa que los excluye y silencia como referentes a ser estudiados.

Para efectos del análisis que se propone, debido a la carga negativa que tienen estos ciclos de luchas fratricidas y la consecuente dificultad que representa su explicación en espacios educativos, las narrativas históricas y escolares que se han construido alrededor de esta confrontación violenta se han entendido desde la categoría difficult histories (Epstein y Peck, 2017). Esta noción da cuenta de diferentes formas narrativas, a partir de estas, se busca, en el marco de procesos de aprendizaje, dar explicación a eventos dolorosos, violentos y traumáticos ocurridos en el pasado a nivel regional, nacional o global.

Asimismo, aunque se reconoce que las narrativas escolares en torno a procesos traumáticos y violentos no solo están definidas por la historia a enseñar -esto es, el currículo oficial-, y que por tanto existen otras variables a tener en cuenta -la agencia de los estudiantes, la agencia de los profesores y la influencia de los medios de comunicación- (Abril, 2015; Ahonnen, 2012, 2014; Barton y McCully, 2010; Borries, 2011, 2016; González, 2017; Henríquez y Muñoz, 2017; Kokkinos, 2011; Valls, Parra y Fuentes, 2017), debido al alcance y naturaleza del problema de investigación propuesto se definieron como fuentes de indagación sujetas a un análisis de contenido de tipo cualitativo (Krippendorff, 1990): 1) la normatividad que reguló de 1903 a 1984 la enseñanza de la historia en el sistema escolar, y; 2) algunos manuales escolares de alta difusión y circulación en el período objeto de estudio. En este sentido se propone un tipo de análisis que en otros contextos nacionales ha sido fructífero para entender la construcción de identidad en contextos de conflictividad social y cultural (Ahonnen, 2014; Chaupis, 2015; González, 2001-2002, Villalón y Pagès, 2015)

\section{La enseñanza de la historia en contextos de posguerra: el caso de la Guerra de los Mil Días}

A pesar que en Colombia durante el siglo XIX la tarea de enseñanza que desarrolló la escuela estaba en función de la patria y la república, "fue solamente a comienzos del siglo XX cuando se comenzó a configurar un principio nacionalista del pasado" (Álvarez, 2007: 385). En otras palabras, los procesos de selección del qué, cómo y para qué se debía recordar -los que fueron definiendo una memoria oficial-, comienzan a afinarse curricularmente con sistematicidad y cobertura a inicios del siglo XX.

Según González (2011), aunque en el siglo XIX se pueden rastrear diferentes disposiciones que integran tempranamente la enseñanza de la historia dentro del plan de estudios oficial, estas no aseguraron en la práctica una efectiva implementación por parte de las escuelas de las orientaciones dadas. Así pues, en el caso específico de la Historia

a pesar de su inserción temprana en el plan de estudios para las escuelas de parroquia y cantón en 1826 por parte del general Santander, su inclusión como materia obligatoria en todas las universidades de la república en el año 1836, 
o su reconocimiento como materia en el derecho legislativo que organizó los colegios nacionales durante el gobierno del General José Hilario López en 1850, no se garantizó de este modo su presencia en la escuela ni mucho menos la popularización de la historia patria (González, 2011: 22).

Esta noción de Historia Patria y el principio nacionalista acerca del pasado que impulsa, cobraron relevancia para el Estado colombiano en un contexto histórico de agitación y tensión sociopolítica muy fuerte que puso en entredicho la viabilidad del proyecto de nación a inicios del siglo XX. Los efectos devastadores prácticos y simbólicos que trajo consigo la Guerra de los Mil Días (1899-1902) ${ }^{3}$ y la consecuente pérdida del territorio de Panamá tras su separación (1903), impulsaron al Estado a definir como mecanismo de generación de unidad, identidad, orden y control sociopolítico, varias políticas educativas que buscaban definir con claridad aquello que se debía recordar e implícitamente olvidar, hecho que impactó directamente cómo se entendía la enseñanza de la historia. Desde esta perspectiva se formuló la ley 39 de 1903 -Ley orgánica de instrucción- y su decreto reglamentario 491 de 1904, documentos normativos en los que se da relevancia y centralidad por primera vez a la Historia Patria. Por ejemplo, se contemplaba que

Será deber primordial en ellos [los directores] despertar y avivar el amor a la patria, por una educación especial, que consista en excitar entusiásticamente el sentimiento de los niños a favor del país natal. Exposiciones frecuentes sobre las bellezas de la patria, sobre sus fastos gloriosos, sobre los hombres que le han ilustrado, inspira a los niños legitimo orgullo de pertenecer a un país que tiene tantos títulos a su afecto, y suscitan en ellos el entusiasmo patriótico (Congreso de la República [CR], 1903).

De esta forma, la escuela impulsó y fundamentó ciertos ejercicios de gubernamentalidad (Álvarez, 2013; Arias, 2015), constituyéndose en el espacio ideal en el que se difundió el relato nacionalista, ya que el Estado colombiano entendió que los niños y los jóvenes al ser potenciales ciudadanos se constituían en sujetos claves de intervención ideológica (Muñoz, 2103). Este relato nacionalista y patriótico, en medio de un contexto social de incertidumbre y quiebre de los lazos de identidad colectivos, buscó generar puntos de anclaje en donde se refundara un discurso de pertenencia colectiva que permitiera a la sociedad colombiana asumirse desde un criterio de "unidad". Por esta razón, se construyó un relato histórico -Historia Patria- que buscó dar coherencia y legitimidad al proyecto de las élites gobernantes, afincado en la institución y exaltación de héroes, acontecimientos y hechos que pretendían homogeneizar la experiencia histórica y cultural de diversos sujetos, pueblos y territorios desde un mismo horizonte discursivo-explicativo.

En este sentido, se difundieron en las escuelas muchos mitos fundacionales del Estado-nación colombiano, los que se presentaron como verdades irrefutables que debían ser memorizadas e interiorizadas por los futuros ciudadanos, y, que finalmente, sirvieron para evadir el análisis de las historias difíciles que estructuraban en la práctica las realidades políticas y sociales del país. Por medio de estos mitos y la exaltación de héroes, se fundamentó un ejercicio de transmisión y formación de una memoria oficial que privilegió el abordaje de acontecimientos con una fuerte carga moral, los que, desde intereses políticos ubicados en un presente desestabilizado por la crisis social, política y económica, con frecuencia fueron tergiversados y descontextualizados.

Estos intereses políticos finalizada la Guerra de los Mil Días, hicieron que se concibiera "en la educación el modelo nacional de progreso con el fin de evitar nuevas confrontaciones y elevar el nivel de riqueza, el espíritu y el carácter nacional por la senda de una civilización moderna y cristiana" (Ghotme, 2010: 162). Objetivos que en su momento obturaron por completo la posibilidad de entender realmente las causas de la desarticulación política y las constantes luchas fratricidas que marcaron la historia de Colombia durante todo el siglo XIX. Así pues, la enseñanza de la historia comenzó a ser "inspirada por un espíritu de reconciliación que quería uncir el pasado a una visión promisoria y optimista del progreso" (Colmenares, 1991: 125); espíritu desde donde se fue definiendo una seudoneutralidad analítica del pasado que instauró una "ley del silencio" y 
un olvido acrítico frente a los fenómenos sociales conflictivos que acompañaron el proceso de formación del Estado-Nación colombiano.

La escuela desde esta óptica promovió desde la enseñanza de la Historia Patria el "olvido" de ciertos acontecimientos y agencias humanas que ponían en duda el proyecto de unidad nacional. Paralelamente, mitificó otros instaurándolos como referentes incuestionables de identidad, los que claro está, eran funcionales a las elites. En este contexto el proyecto educativo impulsado desde la enseñanza de la Historia Patria encuentra en la Academia Colombiana de Historia -en adelante ACH- y la circulación de manuales escolares dos piezas clave de su accionar, las que estarán íntimamente ligadas en la primera mitad del siglo XX.

La ACH fue creada mediante el decreto 1808 dictado por el poder ejecutivo en 1902, documento que la estableció como "Academia Oficial" y "cuerpo consultivo del gobierno" -siendo ratificada como tal por la Ley 24 de 1909- designaciones con los que concentró el poder de decisión frente al qué, por qué y cómo enseñar la historia de Colombia. En sus manos estuvo la construcción de un modelo ideal de patriotismo el que estuvo sujeto a su constante supervisión. De ahí que tuviese injerencia en la producción y revisión de los manuales escolares de historia que se utilizaban en las escuelas del país.

Los manuales fueron claves en la difusión de la Historia Patria y por tanto de la memoria oficial, porque, en gran medida, ayudaron a definir y delimitar las temáticas, conceptos y metodologías que debían fundamentar los procesos de enseñanza de la historia en el país y consecuentemente la salvaguarda de la identidad nacional. Por consiguiente, su intención principal era la de

promover el ideario de la unidad nacional a partir de referentes como el territorio, la ley (como fuente de orden y gobernabilidad de los sistemas republicanos), la religión católica como moderadora del alma nacional; la lengua castellana como vaso comunicante entre la población y la iconografía patriótica (Herrera, 2007: 57).

Todos estos referentes fueron transmitidos desde una escritura cerrada y doctrinaria que no daba margen a cuestionamientos, preguntas $\mathrm{o}$ análisis críticos tanto del pasado como del orden social vigente (Herrera, Pinilla y Suaza, 2003). El ejemplo más claro de este tipo de esquema argumentativo se encuentra en el texto Historia de Colombia de Henao y Arrubla (1911), manual que por más de cinco décadas fue la fuente y referente central a partir del que escolarmente se explicó la Historia de Colombia. Este ejemplar en el marco de los eventos conmemorativos del centenario de la independencia, fue seleccionado como ganador de un concurso que buscaba premiar un texto de historia patria in extenso -dirigido a la enseñanza secundaria- y un compendio de la misma -dirigido a la enseñanza primaria- con el fin de adoptarlos como textos oficiales y difundirlos por todo el país conforme con lo establecido en el decreto 491 de 1904 acerca de instrucción pública. Uno de los criterios generales principales que se fijaron en dicho concurso era el de constituirse en una propuesta "alternativa" que superara el visón "sesgada" y partidista de los textos vigentes en ese momento (Melo, 2010; Pinilla, 2003).

Luego de ser declarados ganadores por jurados que fueron seleccionados por la $\mathrm{ACH}$ y ser aprobados por la Arquidiócesis de Bogotá ${ }^{4}$, mediante el decreto 963 de 1910 los textos de Henao y Arrubla fueron adoptados oficialmente, declarándose su vigencia hasta la convocatoria de un nuevo concurso -el que nunca se dio-. Estos textos potenciaron en la escuela principios explicativos acerca del origen de la nación y su posterior desarrollo, a partir de un esquema narrativo que buscaba "conciliar posiciones ideológicas $(. .$.$) en torno a una visión de proyecto de$ Estado-Nación apoyada en la visión conservadora (empresa de cristianización y misión civilizadora europea de los períodos de conquista y Colonia) y la liberal (necesidad del imperio de la ley)" (Colmenares, 1991: 126). Por tanto, lo importante en esta propuesta editorial era alinearse con el espíritu de reconciliación nacional que se impulsaba desde los gobernantes de la época, sin importar que esta búsqueda estuviese definida por la manipulación y tergiversación del pasado (Alarcón, 2013). Lo fundamental entonces era promover una "cultura de la paz" mediante la transmisión de verdades reveladas que, al reducir la heterogeneidad interpretativa del pasado, configuraran un sentido homogéneo de identidad dando relevancia a modelos de patriotismo. De allí que en la introducción del texto se declare que el valor de la 
historia en un contexto educativo se comprueba si esta

cultiva eficazmente la memoria y la imaginación; ilustra la razón y la conciencia, y fortalece la voluntad; da variadas y múltiples lecciones instructivas y recreativas; pone al futuro ciudadano en capacidad de formar opiniones precisas y sanas, para quedar a cubierto de las influencias dañinas de la ignorancia y la credulidad que oscurecen la verdad y comprometen la paz (Henao y Arrubla, 1967: 22).

Pese a que posteriormente durante la llamada República liberal ${ }^{5}$ emergieron nuevos discursos alrededor de la enseñanza de la historia impulsados por nuevas construcciones epistémicas acerca de este saber escolar, la profesionalización de los maestros (Arias, 2015), un interés inusitado por el tiempo presente, y hasta el cambio de la denominación formal del saber histórico en el currículo $^{6}$ (Álvarez, 2013), el texto escolar de Henao y Arrubla durante este período de "cambio" siguió definiendo la reflexión de identidad nacional desde el esquema de la Historia Patria, tanto de su uso generalizado en las escuelas como de su influencia decisiva en posteriores textos que comenzaron a circular. Con ello, durante toda la primera mitad del siglo XX, la enseñanza de la historia, los manuales escolares que apoyaban su ejercicio y la escuela en general -al menos desde el currículo oficial-, evadieron el análisis de la conflictividad sociopolítica que había marcado el devenir histórico del país; conflictividad que lejos de ser superada por el espíritu reconciliador impulsado por las elites a inicios del siglo XX, se había complicado aún más y desembocado en una nueva lucha fratricida que se ahonda en la década de 1940 y se extiende por toda la década de 1950 -La Violencia-.

\section{La reafirmación de la historia patria como referente de identidad: el caso del período de "La Violencia"}

El ciclo de confrontación fratricida que define lo que académicamente se ha denominado como el período de "La Violencia"7, nuevamente fue evadido como tema de estudio y conforme con la fórmula utilizada décadas atrás por los gobiernos conservadores para solventar las heridas de la Guerra de los Mil Días, impulsó el fortalecimiento de la Historia Patria como único referente a ser tenido en cuenta para entender la historia de Colombia. Así pues, tras los hechos del 9 de abril de $1948^{8}$ y las tensiones sociopolíticas que se derivan de este acontecimiento, el gobierno conservador de la época liderado por Mariano Ospina Pérez mediante la expedición de varios decretos y reglamentaciones curriculares, intensificó la enseñanza de la historia a partir de la base de retomar la Historia Patria como su eje central (Acevedo y Samacá, 2012; Arias, 2015; Betancourt, 2007; Ghotme, 2010; González, 2014; Rodríguez, 2009; Velandia, 2001). Con ello se buscaba fundamentalmente "(...) preparar la población para una vida cívica, comprometida con el respeto de la legalidad, la moralidad y la autoridad" (Rodríguez, 2009: 136); valores que se consideraban centrales en un momento de caos social como el que se estaba viviendo. Dicha inestabilidad social es la que el Estado esgrimió como excusa para intensificar la enseñanza de la historia de Colombia en el currículo oficial. Por ejemplo, en el decreto 2388 de 1948 se afirma:

los graves acontecimientos que en los últimos tiempos han agitado a la República han puesto de manifiesto, una vez más y con caracteres de grande apremio, que el estudio concienzudo de la historia patria y la práctica de las virtudes cívicas por todos los hijos de Colombia deben ser preocupación permanente y desvelada del Gobierno (Presidencia de Colombia [PC], 1948).

Ello, según el Estado, explicaba y exigía el establecimiento de ciertas acciones concretas que finalmente impactaron las prácticas escolares derivadas de la enseñanza de la historia. En consecuencia se establecieron lineamientos moldeados desde el espíritu de la Historia Patria que contemplaron desde aspectos administrativos -una selección más rigurosa de los profesores de Historia; la modificación de la carga horaria asignada a la historia; el fortalecimiento de procesos de supervisión y vigilancia; el otorgamiento de premios y estímulos tanto a maestros como a estudiantes-, hasta la instauración o ampliación de prácticas escolares de carácter obligatorio: 
establecimiento de fechas particulares para exaltar a fundadores y grandes promotores de la nacionalidad; la exaltación de las figuras de Bolívar y Santander por medio de la ubicación de sus retratos en los establecimientos educativos de todo el país; la adopción por parte de las escuelas de un patrono cívico seleccionado entre los fundadores de la Patria; el fortalecimiento del culto a la bandera, el himno y el escudo nacionales.

Asimismo, dentro de las disposiciones más importantes que se establecieron en la búsqueda de reposicionar la Historia Patria como eje central de la educación impartida en las escuelas se encuentran: la revalidación de la ACH como instancia reguladora de la enseñanza de la historia y la centralidad de los manuales escolares en el proceso de "transmisión" de esta. Por consiguiente, los manuales escolares con el direccionamiento y aprobación de la $\mathrm{ACH}$ debían fortalecer los principios de la memoria oficial que se fueron estructurando a inicios del siglo XX y producir un relato histórico oficial acerca del 9 de abril que fuera funcional a la idea de "orden" y "estabilidad" sociopolítica. El objetivo era establecer una estrategia discursiva de carácter masivo que coayudara a detener la proliferación de la confrontación bipartidista y la posible generación de una revolución social. Esta tarea se desarrolló desde dos procedimientos generales: el olvido, mediante del no tratamiento del problema de la Violencia como un proceso complejo en el que confluían problemáticas cuyas raíces estaban en el pasado y se extendían en el presente, y, la tergiversación y construcción de una lectura limitada de los antecedentes, desarrollo y consecuencias del 9 de abril.

Manuales escolares como los de Ramón Hermano Justo (1964), Julio César García (1969) y, Rafael Granados $(1972,1976)$ que alcanzaron a tener una amplia difusión en sus numerosas ediciones -se publicaron hasta la década del ochenta-, abordaron el tema de la Violencia a partir de este esquema de omisión y tergiversación. Dentro de las omisiones más importantes se encuentran: los procesos de confrontación y tensión social que se vivieron durante los años cuarenta; la centralidad del movimiento gaitanista y el legado político de Gaitán; la lucha bipartidista; la organización social y resistencia que se generó durante y luego del 9 de abril de 1948; la ola de Violencia que azotó el país durante el gobierno de Laureano Gómez a inicios de la década del cincuenta, etcétera.

Dichas omisiones se definieron desde una estructura expositiva lineal de la historia de Colombia que, además de concentrar la mayor parte de su explicación en los períodos de la "Colonia" y la "Independencia", dio cuenta de la historia del siglo XX mediante una descripción sintética y limitada de los logros económicos, políticos y sociales en cada uno de los períodos presidenciales. A partir de este esquema expositivo, por ejemplo, las ideas políticas de personajes como Jorge Eliecer Gaitán quedaron invisibilizadas.

Por otra parte, en estos manuales, los hechos con algún tipo de relación con la Violencia se desarrollaron sin mayor profundidad analítica desvirtuándolos como casos aislados e inconexos que fueron superados. Es el caso del tratamiento que se le da al 9 de abril de 1948, su "presencia" como tema a ser recordado, además de ser desarrollado de una forma subsidiaria, secundaria y dependiente de la descripción del período presidencial de Mariano Ospina Pérez, se materializó desde una perspectiva de corta duración que limitó su alcance en el tiempo y el espacio. De ahí que los elementos que se resaltaron fueran la influencia comunista en los hechos -llevando al plano internacional la causa del asesinato del caudillo liberal- y la barbarie de la insurrección que trajo consigo la muerte de Gaitán. Por ejemplo, en el texto "Historia General de Colombia" se afirma que

fue el comunismo internacional el autor intelectual de la tragedia, la cual obedeció, no a móviles de política colombiana, sino a motivos de orden universal. Las masas bogotanas sirvieron de instrumento ciego para la realización de planes, a los cuales ellas mismas eran completamente extrañas. El asesinato de Gaitán no fue un hecho concomitante ocurrido casualmente, sin conexión alguna con el plan de sabotaje a la Conferencia Panamericana, sino que constituyó la base fundamental de aquel plan y fue la condición sine qua non para hacerla posible (Granados, 1976: 510).

Luego de proponer como verdad absoluta la responsabilidad exclusiva del comunismo 
internacional en la muerte de Gaitán -explicación rebatida y cuestionada por las investigaciones académicas respecto del tema-, los manuales se enfocaron en resaltar el espíritu de barbarie desde el que se desarrolló el 9 de abril, en contraposición de los valores morales, cristianos y cívicos del proyecto nacional. Se afirma entonces que

La muchedumbre armada, obedeciendo a la voz de mando pronunciada desde las emisoras revolucionarias se entregó al saqueo y al incendio, con preferencia de templos, colegios de religiosos y edificios públicos: de esta suerte, fueron entregados a las llamas la iglesia colonial del Hospicio, el convento de la Inmaculada Concepción, el instituto de la Salle con su valiosísimo museo, la Nunciatura, el Palacio arzobispal, el Ministerio de Gobierno, el Palacio de Justicia, el de San Carlos, la gobernación y el edifico de "El Siglo"; las imágenes sagradas fueron despedazadas y el comercio bogotano fue entregado al saqueo (Granados, 1976: 512-513).

A partir de los mismos principios explicativos generales, en el texto "Curso Superior de Historia de Colombia" se afirma

El 9 de abril de 1948 fue asesinado en Bogotá el doctor Jorge Eliecer Gaitán. La noticia de la muerte del caudillo liberal produjo un desbordamiento de matanza, rapiña e incendios en la capital y en otras ciudades y poblaciones del país, como no se había registrado antes ni aún en las más sangrientas guerras civiles, que amenazó borrar el pasado histórico y cuanto constituye el patrimonio moral de la República (García, 1969: 316).

Estas descripciones y acentos específicos fundamentaron en los manuales escolares analizados los argumentos de cómo se explicó el 9 de abril de 1948. Con ello explícitamente se hace omisión a los procesos de organización y resistencia social que se dan antes, durante y luego del 9 de abril en sectores tanto urbanos como rurales en buena parte del territorio nacional (Rodríguez, 2009). Procesos con mayor peso histórico que los actos de destrucción que enmarcaron esa jornada.

Sumado a ello, la estrategia narrativa empleada en los textos buscó completar el ocultamiento de la importancia de Gaitán y sus ideas a partir de la exaltación de personajes que encarnaban un modelo contrario al suyo y que, a la postre, no tuvieron una importancia histórica decisiva como sí la tuvo el caudillo liberal. De esta forma se generó una exaltación de la figura del presidente Mariano Ospina Pérez, el que fue tratado como héroe de la patria y personaje central del 9 de abril. Se utilizaron para ello muchas exageraciones que buscaban mitificar su papel, resaltando hechos no comprobados y por fuera de toda lógica, como el que explica su intento de asesinato en medio del desarrollo de los actos vandálicos desatados esa fecha, el que según un manual fue impedido por "dieciséis heroicos soldados, comandados por denodados jefes, que durante más de tres horas contuvieron el empuje violento de diez mil asaltantes" (Granados, 1976: 512); o el que reclama su relevancia al considerar que su "actitud (...), en aquella hora de zozobra, halla pocos similares en la historia" (Granados, 1976: 513).

En este sentido, una tarea prioritaria para los autores de los manuales que se producen luego del 9 de abril, fue resaltar el valor patriótico de la clase dirigente -conservadores y liberalesquienes, según ellos, evitaron el colapso del Estado-nación. De allí que se buscara en algunos casos manejar un discurso "reconciliador" que permitiera reconocer la labor de la dirigencia de los dos partidos. Por ejemplo, en uno de los manuales de mayor difusión se resalta que "la heroica entereza del Doctor Mariano Ospina, la lealtad y el valor del ejercito y la comprensión patriótica del Doctor Darío Echandía salvaron a Colombia de una catástrofe definitiva" (García, 1969: 316).

Con la invención de nuevos "héroes" de la patria y su papel decisivo en el aseguramiento del orden democrático, los manuales de historia de Colombia que circularon hasta la década del ochenta instauraron discursivamente una memoria oficial en la que la Violencia como fenómeno social fue desterrada del territorio nacional. Visión que en contraste con la realidad no tenía fundamento alguno. Por consiguiente, al declararse explícitamente "el triunfo del orden obtenido el 9 de Abril (...), el triunfo de la civilización cristiana sobre la barbarie" (Granados, 1976: 514), 
los posteriores fenómenos de recrudecimiento de la violencia -que sobrevinieron luego de esta fecha fueron definidos como eventos fortuitos y aislados que se estructuraron desde “(...) una violencia incausada, en la que no tienen relevancia alguna el escenario social ni el contexto nacional" (González, 2014: 37).

Así pues, fenómenos como la conformación de guerrillas y el establecimiento de la lucha armada entre insurgentes y fuerzas del Estado que se ha desarrollado desde la década del sesenta del siglo XX, siguieron estando definidos por el currículo oficial a partir del olvido y una presencia ausente que le restó centralidad e importancia. Posicionamientos que fueron reafirmados desde el esquema explicativo que estructuraba la difusión de la Historia Patria.

Contrario a los discursos escolares oficiales, la producción investigativa en el país comenzó a partir de las décadas de 1950 y 1960 a desarrollar posturas críticas de ese fenómeno que denominó "La Violencia", introduciendo con ello nuevas temáticas y problemas de estudio que no habían sido tratados pese a su importancia en la comprensión de la historia del país. Según Colmenares (1991), los trabajos investigativos desarrollados en ese período generan una apertura fundamental en los procesos de comprensión e interpretación de la historia de Colombia, al cuestionar los relatos impulsados por la memoria oficial. Sin embargo, estas nuevas formas de analizar críticamente el pasado del país, no lograron impactar en ese momento las prácticas y discursos desde los cuales se sustentaban los procesos de enseñanza escolar de la historia. En este sentido, tanto los planes de estudio como las propuestas de los textos escolares de la época-que eran reediciones de los manuales de la primera mitad del siglo XX- mantuvieron intacto el ideario homogeneizador de la Historia Patria, reproduciendo procesos de olvido acrítico en torno a problemas históricos centrales como el que constituye "La Violencia".

Posteriormente, a mediados de la década del setenta del siglo XX y en el marco del inicio de un proceso de reforma institucional y curricular de la educación colombiana, se comenzaron a establecer una serie de transformaciones parciales en los textos escolares de historia; cambios que, aunque no alcanzaron una profundidad suficiente que rompiera definitivamente con las líneas explicativas propuestas por la Historia Patria, proponían un 'tratamiento menos 'ideologizado' de los contenidos, con un evidente interés por plantear un tipo de contenidos que intenta superar la presentación lineal de los acontecimientos políticos" (Gómez, 2001: 163). Esta búsqueda de renovación se apoyó en diferentes procesos que fueron confluyendo entre sí: el proceso de profesionalización de las ciencias sociales en el país, el desarrollo de nuevas pautas pedagógicas y, fundamentalmente, la convergencia de corrientes historiográficas derivadas del Marxismo y la escuela de los Annales en el pensamiento historiográfico colombiano.

Esta concomitancia le da origen al movimiento de la Nueva Historia, el que será decisivo en las décadas del ochenta y noventa del siglo XX en el debilitamiento de la Historia Patria como paradigma hegemónico, posicionando disciplinar y escolarmente temas que antes no eran registrados ni analizados, y cuestionando otros que se presentaban naturalmente como verdades absolutas desde la academia y la escuela (Ibagón, 2016). Se configuró de esta forma un proceso de "tránsito entre los postulados centrados en promover el sentimiento patriótico y aquella tendencia que quería darle al conocimiento social mayor impacto en la construcción de la democracia y el desarrollo" (Acevedo y Samacá, 2012: 228).

En medio de este proceso de tránsito, a partir de la segunda mitad de la década del ochenta del siglo XX se generó un escenario de confrontación ideológica y pedagógica entre dos posiciones que entendían de forma diferente, tanto la historia de Colombia como su enseñanza. Esta confrontación fue denominada la batalla de los manuales ${ }^{9}$ (Colmenares, 1991). En uno de los sectores en disputa se encontraban autores de textos escolares que amparados en el movimiento de la Nueva Historia planteaban una renovación de los principios desde los que tradicionalmente se estructuraba la historia del país (Rojas y Hurtado, 1984; Kalmanovitz y Duzán, 1986; Melo y Díaz, 1989), en el otro sector se encontraba la Academia Colombiana de Historia, institución que profesaba la necesidad de defender a toda costa la identidad que se impulsaba mediante la enseñanza de la Historia Patria.

Esta confrontación de ideas permitió en el mediano plazo impulsar en los libros de texto una "renovación" considerable de algunas temáticas y contenidos, a partir del fortalecimiento de enfoques analíticos centrados en lo económico y social, 
que establecieron una alternativa "crítica" a una historia "política" que durante todo el siglo XX se había sustentado en la narración de las proezas de grandes héroes y acontecimientos puntuales, los que no daban cuenta de procesos más amplios y complejos. De forma complementaria la presencia más fuerte de dimensiones pedagógicas que comenzaron a ser decisivas en la posibilidad tanto de la renovación de los contenidos como de las formas de abordarlos al interior del aula de clase, se configuró como un avance en la construcción de los libros de texto de la época. En este punto es clave la influencia del movimiento pedagógico ${ }^{10}$ y su reivindicación de la pedagogía como campo de conocimiento en el país.

Tal vez uno de los elementos más significativos que van a fortalecer las transformaciones de la enseñanza escolar de la historia del país -al menos discursivamente- es la postulación legal de la idea de integración que fundamenta la aparición de las Ciencias Sociales dentro del currículo oficial. Esta denominación de ciencias sociales y el tema de la integración se pueden rastrear a inicios de la década del setenta con las reformas que emprendieron la modernización del sistema educativo del país y los numerosos debates académicos que evidenciaron

la proliferación y complejidad de las fuerzas de poder que pugnaban por redefinir la función que debía cumplir su enseñanza en la escuela, sobre todo la historia y la geografía, que hasta entonces habían estado alinderadas con la cívica y la urbanidad en la construcción de un ciudadano nacional (Cortés, 2012: 194).

Sin embrago, es con el decreto 1002 de 1984 expedido por el Ministerio de Educación Nacional, con el que se inicia una operativización -lenta- de la propuesta, definiéndose dentro del plan de estudios la existencia de un área común para la educación básica y media vocacional llamada Ciencias Sociales (Guerrero, 2011; Ibagón, 2016). Dicho cambio impactó considerablemente la propuesta de los textos escolares, los que en buena parte la incluyeron como su soporte epistemológico (Sánchez y Bolívar, 2015).

Pese a que la historia fue entendida como el eje central de la integración, en el escenario educativo se comenzaron a desarrollar desde esta última idea procesos de reacción en contra de la historia tradicional; procesos que se sustentaron en la implementación de referentes epistemológicos e historiográficos alternos y elementos didácticos y pedagógicos que revitalizaron la necesidad de reflexión alrededor de la enseñanza de la historia ahora en el marco de las Ciencias Sociales. Estos nuevos posicionamientos permiten a algunos textos escolares -como el de Rojas y Hurtado (1984), Kalmanovitz y Dúzan (1986), Melo y Díaz (1989) - reconocer problemas de estudio y agentes históricos asociados a la violencia (campesinos, sindicatos, guerrillas terratenientes y personalidades políticas como la que representa Gaitán) desde una perspectiva reivindicativa. En este sentido, "donde los autores de la primera generación [de textos escolares] habían encontrado subversión, bandoleros, chusma y delincuentes, los ochenta empezaron a encontrar rebeldes, guerrilleros e insurgentes" (González, 2014: 41).

Sin embargo, el peso de una larga tradición educativa que inscribía la enseñanza de la historia exclusivamente a partir del culto a los héroes y la memorización, sumada a la falta de claridad operativa de la propuesta y la lentitud en la construcción de un marco curricular que solo vio la luz cinco años después, imposibilitaron en este primer momento desarrollar transformaciones de fondo. De ahí que, pese a que la idea de la integración fue acogida por diversos actores escolares entre ellos algunas editoriales, gran parte de los mitos y estereotipos históricos con los que tradicionalmente se daba cuenta del pasado de Colombia siguieron siendo la base constitutiva de los textos escolares que se comenzaron a producir posteriormente.

En este sentido, este impulso "renovador" aunque posicionó nuevos discursos y explicaciones acerca de la historia nacional, no logró en definitiva consolidar una revisión crítica desde el currículo prescripto de ese pasado conflictivo que marca el presente del país. Revisión que, hoy más que nunca, es fundamental si se tiene en cuenta la reciente firma del acuerdo de paz entre el Estado colombiano y uno de los actores más importantes del conflicto armado -no el único-: la guerrilla de las FARC.

\section{Conclusiones}

La enseñanza de la historia que se impulsó desde el currículo oficial a lo largo del siglo XX 
en Colombia, impidió y obstaculizó una revisión crítica de la conflictividad sociopolítica interna que caracterizó -y sigue caracterizando- el devenir histórico del país. De esta forma, a diferencia de temas de estudio como el de la Independencia política conseguida a comienzos del siglo XIX, cuyo eje explicativo escolar se centraba en el aprendizaje de un caso afirmativo de identidad que representaba gloria y satisfacción, las guerras civiles declaradas y "no declaradas" que definen el comienzo y desarrollo del siglo XX en Colombia, al ser procesos con una carga negativa, se fueron constituyendo en temas que el currículo oficial en su momento silenció o tergiversó.

En este sentido, el esquema narrativo y explicativo de la llamada Historia Patria se configuró como uno de los mecanismos más importantes con los que el sistema escolar evadió el análisis de las causas y consecuencias de los diferentes ciclos de violencia por los que fue atravesando Colombia durante el siglo XX. De esta forma, a partir de la idea de "unidad" y "reconciliación" se fue definiendo una política del olvido que impidió que la escuela fuese un escenario de reflexión asertiva para comprender el por qué de varias confrontaciones que han desangrado al país.

Dicha política del olvido tuvo en las orientaciones curriculares y los manuales escolares piezas clave que ayudaron a la construcción de narrativas orientadas a propiciar escenarios de unidad nacional -fundamentados en intereses políticos e ideológicos particulares-, las que fueron a lo largo del siglo XX aplazando indefinidamente una lectura crítica escolar de las problemáticas y conflictos sociopolíticos que en la práctica estaban fracturando al país. Este aplazamiento de un estudio crítico del pasado distante y reciente de Colombia sigue en la actualidad marcando en buena parte el esquema de funcionamiento de la enseñanza de la historia, hecho que se vuelve complejo en la actualidad, en un contexto de transición sociopolítica definido por la firma de un acuerdo de paz histórico.

En consecuencia, la experiencia de la difusión escolar del olvido acrítico que ha caracterizado a la enseñanza oficial de la historia de Colombia se configura como un punto de referencia inevitable a la hora de entender y definir críticamente los retos a los que se ve abocada la enseñanza de la historia hoy. Retos en los que se incluye la construcción de una memoria histórica que permita reconocer los problemas que han definido un conflicto complejo, el que, con el tiempo, ha mutado y sigue mutando. Es a partir de una voluntad política y pedagógica que se puede impulsar la revisión y reconfiguración de ese esquema, ayudando así a generar y consolidar las bases del inicio de un proceso de reconciliación profundo, el que tenga en la escuela un espacio fundamental de acción.

\section{Agradecimientos}

Agradezco el apoyo brindado por el Departamento de Historia de la Universidad del Valle y al Grupo de Investigaciones Históricas en Política, Economía y Educación -IHEPE- por el acompañamiento académico que realizó al proceso de ejecución de la propuesta investigativa que da origen al presente artículo.

\section{Referencias Citadas}

Abril, D.

2015 "Ciudadanía, educación y complejidad: miradas desde la ecopedagogía". Diálogo Andino. Revista de Historia, Geografía y Cultura Andina 47: 95-104.

Ahonnen, $\mathrm{S}$.

2014 "Education in post-conflict societies. Historical Encounters". A Journal of historical consciousness, historical cultures and history education, 1: 75-87.

Ahonnen. S.

2012 Coming to terms with a dark past. How post-conflict societies deal with history. Peter Long, Frankfurt.

Acevedo, A. y Samacá, G.

2012 "La política educativa para la enseñanza de la Historia de Colombia (1948-1990): de los planes de estudio por asignaturas a la integración de las ciencias sociales". Revista Colombiana de Educación, 62: 221-244.

Alarcón, L.

2013 "Representaciones sobre la independencia en los manuales de historia de Colombia". Investigación \& Desarrollo. Revista Latinoamericana de Ciencias Sociales y Desarrollo Humano, 21: 342-370.

Álvarez, A.

2013 Las ciencias sociales en Colombia. Genealogías pedagógicas. IDEP, Bogotá.

Álvarez, A.

2007 Las Ciencias Sociales en el currículo escolar. 19301960. Tesis para optar al grado de Doctor en educación, UNED, Madrid. 
Arias, L.

2015 "Continuidades y discontinuidades de la historia escolar en Colombia”. Pedagogía y Saberes, 42: 31-41.

Barton, K. y McCully, A.

2010 "You can form your own point of view internally persuasive dialogue in Northern Ireland student's encounters with history". Teachers Colleged Record 112: 42-181.

Betancourt, A.

2007 Historia y Nación: Tentativas de la escritura de la historia de Colombia. La Carreta, Medellín.

Borries, B.

2016 Jovens e consciência histórica. W.A Editores, Curitiba.

Borries, B.

2011 "Coping with burdening history". In Historicizing the uses of the past, Scandinavian Perspectives on History Culture. Historical Consciousness and didactics of History related to World War II, Bjerg, H.; Lenz, C. and Thostensen, E. (Eds.), pp. 283-319. Transcript, Bielefeld. Chaupis, J.

2015 "Los textos escolares en conflicto con la subalternidad: La Guerra del Pacífico en las aulas". Diálogo Andino. Revista de Historia, Geografía y Cultura Andina 48: 99-108.

Colmenares, G.

1991 "La batalla de los manuales en Colombia". En Latinoamérica: enseñanza de la historia, libros de texto y conciencia histórica, Riekenberg, M. (Ed.), pp. 122134. Alianza Editorial/FLACSO/Georg Eckert Institut, Buenos Aires.

Cortés, R.

2012 Prácticas de ciudadanización en la escuela contemporánea: Colombia 1984-2004. Tesis para optar al grado de Doctora en Educación, Universidad Pedagógica Nacional, Bogotá.

Epstein, T. y Peck, C.

2017 Teaching and learning difficult histories in international contexts. A critical sociocultural approach. Routledge, New York.

Ghotme, R.

2010. "Nación y heroísmo en Colombia, 1910-1962". Revista de Relaciones Internacionales, Estrategia y Seguridad 5: 161-191.

Gómez, M.

2001 "La revolución Francesa en los manuales escolares colombianos de ciencias sociales e historia: estructura temática y contexto educativo". Educación y Pedagogía 13: $145-167$.

González, I.

2014 "La violencia contada a los escolares. Conflicto social y memoria en los manuales educativos del siglo XX". Análisis Político, 81: 32-48.

González, M.

2011 La configuración histórica del saber pedagógico para la enseñanza de la Historia en Colombia, trazos de un camino: 1870-2010. Tesis para optar al grado de Maestría en Historia, Universidad Javeriana, Bogotá.

González, M. P.

2017 "Los saberes históricos escolares como construcción situada y singular". Diálogo Andino. Revista de Historia, Geografía y Cultura Andina, 53: 45-57.
González, S.

2001-2002 "Violencia en los Andes de Tarapacá: una reflexión teórica". Diálogo Andino. Revista de Historia, Geografía y Cultura Andina 20/21: 9-35.

Guerrero, C.

2011 La incidencia de las reformas educativas en la enseñanza de la Historia en Colombia 1973-2007. Tesis para optar al grado de Magíster en Historia, Universidad Nacional de Colombia, Bogotá.

Henríquez, R. y Muñoz, Y.

2017 "Leer y escribir históricamente: los desafíos pendientes de la enseñanza y del aprendizaje de la Historia". Diálogo Andino. Revista de Historia, Geografía y Cultura Andina 53: 7-21.

Herrera, M.

2007 "El memorial de las identidades: entre héroes y villanos. En la busca de sí y de los demás también". Folios, 25: 53-61.

Herrera, M.; Pinilla, A., y Suaza, L.

2003 La identidad Nacional en los textos escolares de ciencias sociales 1900-1950. Antropos, Bogotá.

Ibagón, N. J.

2019 "Conciencia histórica y textos escolares. Un análisis a partir de las concepciones sobre el período de "La Violencia" en Colombia". Revista Encuentros 17: 46-56.

Ibagón, N. J.

2016 Entre ausencias y presencias ausentes. Los textos escolares y el lugar de lo negro en la enseñanza de la historia de Colombia. Universidad Javeriana, Bogotá.

Ibagón, N. y Chisnes, L.

2019 "Narrativas históricas sobre el conflicto armado colombiano y sus actores. Estudio a partir del análisis de textos escolares”. Saber, Ciencia y Libertad, 14 (2): 203-221.

Kokkinos, G.

2011 "History education in relation to the controversial past and trauma". In The future of the past: why History education Matters, Perikleus, L. y Shemilt, D. (Eds.), pp. 34-67. AHDR/UNDP ACT, Chipre.

Krippendorff, $\mathrm{K}$.

1990 Metodología de análisis de contenido: práctica y teoría. Ediciones Paidós, Barcelona.

Melo, J.

2010 La historia de Henao y Arrubla: tolerancia, republicanismo y conservatismo. En Entre el olvido y el recuerdo: iconos, lugares de memoria y cánones de la historia y la literatura en Colombia, De Mojica, S.; Rincón, C. y Gómez, L. (Eds.), Universidad Javeriana, Bogotá.

Muñoz, $M$.

2013 "El ciudadano en los manuales de historia instrucción cívica y urbanidad, 1910-1948". Historia y Sociedad 24: 1-16.

Padilla, A. y Bermúdez, Á.

2016 "Normalizar el conflicto y des-normalizar la violencia: retos y posibilidades de la enseñanza crítica de la historia del conflicto armado colombiano". Revista Colombiana de Educación 71: 219-251.

Pinilla, A.

2003 "El compendio de historia de Colombia de Henao y Arrubla y la difusión del imaginario nacional a comienzos del siglo XX". Revista Colombiana de Educación 45: $2-21$. 
Rodríguez, $\mathrm{S}$.

2009 "El 9 de abril en las políticas de la memoria oficial. El texto escolar como dispositivo del olvido". En Mataron a Gaitán: 60 años, Ayala, C.; Casallas, O. y Cruz, H. (Eds.). Universidad Nacional de Colombia, Bogotá.

Sánchez, N. y Bolívar, R.

2015 "Contenidos de enseñanza en los textos escolares de 1984 al 2010 en Colombia". Pedagogía y Saberes 42: 61-70.

Valls, R.; Parra, D. y Fuertes, C.

2017 "Los temas históricos conflictivos y su abordaje escolar: un ejemplo español”. Clio \& Asociados 25: 8-21.

Velandia, R.

2001 Un siglo de historiografía colombiana: Cien años de la Academia Colombiana de Historia. Academia Colombiana de Historia, Bogotá.

Villalón, G. y Pagès, J.

2015 "La representación de los y las indígenas en la enseñanza de la Historia en la educación básica chilena. El caso de los textos de estudio de Historia de Chile". Diálogo Andino. Revista de Historia, Geografía y Cultura Andina 47: 27-36.

\section{Fuentes primarias}

\section{Leyes y Decretos}

Congreso de Colombia

1903 Diario Oficial 11931. Ley 39 de 1903 (26 de octubre). Sobre instrucción Pública.

Congreso de Colombia

1909 Diario Oficial 13802. Ley 24 de 1909 (28 de septiembre). Por la cual se reconoce carácter oficial a la Academia Nacional de Historia.

Presidencia de Colombia

1910 Diario Oficial 14140. Decreto 963 de 1910 (26 de octubre). Por el cual se adoptan unos textos de enseñanza de la historia de Colombia.

Presidencia de Colombia

1948 Diario Oficial 26779, Decreto 2388 de 1948 (15 de julio). Por el cual se intensifica la enseñanza de la historia patria y se dictan otras disposiciones.
Presidencia de Colombia

1984 Diario Oficial 36615, Decreto 1002 de 1984 (24 de abril). Por el cual se establece el plan de estudios para la Educación Preescolar, Básica (Primaria y Secundaria) y Media Vocacional de la Educación Formal Colombiana.

Vicepresidencia de Colombia

1902 Diario Oficial 11785. Decreto 1808 de 1902 (12 de diciembre). Por el cual se crea la Academia de Historia y antigüedades.

Vicepresidencia de Colombia

1904 Diario Oficial 12122. Decreto 491 de 1904 (3 de junio). Por el cual se reglamenta la Ley 39 de 1903, sobre instrucción pública.

\section{Manuales escolares}

García, J.

1969 Curso Superior de Historia de Colombia. De conformidad con los programas oficiales vigentes sobre la materia, para Cuarto año de Enseñanza Media (Séptima Edición). Voluntad, Bogotá.

Granados, R.

1978. Historia general de Colombia. Lerner, Bogotá.

Granados, R.

1972 Historia de Colombia. La independencia y la República. Instituto Caro y Cuervo: Bogotá.

Henao, J. y Arrubla, G.

1967 [1911] Historia de Colombia para la enseñanza Secundaria. Academia Colombiana de Historia/ Voluntad, Bogotá.

Justo, R.

1964 Historia de Colombia Independencia y República. Stella, Bogotá.

Kalmanovitz, S. y Duzán, S. 1986. Historia de Colombia. El Cid, Bogotá.

Melo, J. y Díaz, G.

1989 Raíces 5. Libros y Libres S.A., Bogotá.

Rojas, J. \& Hurtado, A.

1984 Conozcamos nuestra Historia. Colombia. PIME Editores, Bogotá.

\section{Notas}

1 Es necesario aclarar que estos dos procesos históricos no son los únicos que condensan la conflictividad social y económica del siglo XX colombiano. Sin embargo, a diferencia de otros procesos históricos, estos están directamente relacionados con iniciativas curriculares de intensificación de la enseñanza de la Historia Patria -objeto de reflexión del presente artículo-.

2 Referente que fue puesto en entredicho durante la década de 1980 del siglo XX a partir de la emergencia "renovadora" tanto de posicionamientos curriculares que impulsaron una política de integración de las ciencias sociales como de propuestas editoriales -textos escolares- transgresores del relato oficial.

3 Lucha fratricida de contenido bipartidista iniciada por una facción del liberalismo -Radicales-, que veía en la confrontación armada la única opción de toma efectiva del poder, porque, según su óptica, las presiones burocráticas y persecución política de los conservadores -nacionalistas- en ejercicio del gobierno, habían desvirtuado los caminos democráticos instaurados por la república. Además del elevado número de muertos -100.000 aproximadamente-, la guerra trajo consigo efectos económicos y políticos que generaron una profunda crisis institucional en el Estado colombiano.

4 Debido al concordato establecido entre el Estado colombiano y el Vaticano en 1887, se permitió una vigilancia moral por parte de la Iglesia de todos los documentos que tuviesen algún tipo de relación con la educación. En el caso de los textos escolares la Iglesia tuvo en sus manos la producción o revisión de estos, con el fin que los discursos contenidos en estos dispositivos pedagógicos no fueran en contravía de la doctrina católica. 
5 Período de la Historia de Colombia que marca el fin de la llamada Hegemonía conservadora (1886-1930) a partir de la llegada al poder de Enrique Olaya Herrera, político colombiano de filiación política liberal. La República liberal se extiende de 1930 hasta 1946.

6 Las clases de historia y geografía en el currículo oficial pasan a estar definidas bajo la denominación "Estudios sociales".

7 "La Violencia" es un concepto que dentro de los sectores académicos de las Ciencias Sociales en Colombia ha sido empleado para explicar los complejos procesos políticos y sociales que se vivieron entre las décadas de 1940 y sesenta; décadas en las que el país sufrió una violenta confrontación fratricida que estuvo enmarcada bajo el bipartidismo, la lucha entre colonos y terratenientes, y la aparición del bandolerismo. Para muchos académicos es un período histórico fundamental de Colombia, ya que, en él, se conjugan elementos estructurales y coyunturales de un pasado marcado por la inestabilidad y debilidad del Estado-Nación que se ha proyectado en un presente caótico, donde la violencia y la democracia paradójicamente siguen coexistiendo.

8 Fecha que se convirtió en un hito de la historia de Colombia al marcar un punto de inflexión social y política en torno al problema de la violencia. Con el asesinato del caudillo Liberal Jorge Eliecer Gaitán se exacerba el conflicto.

9 Confrontación de ideas que se desarrolló en el marco de publicaciones de prensa, en el que los bandos en "disputa" establecieron sus líneas de pensamiento alrededor de la renovación o mantenimiento de los principios históricos desde los que se estructuraba la narración de la historia de Colombia.

10 Este movimiento surgió en 1982 como reacción a las reformas curriculares que estaba adelantando el Ministerio de Educación Nacional de esa época, las que buscaban vigilar y controlar política y pedagógicamente la educación en el país. La propuesta del MEN se estructuraba desde un enfoque conductista en el que los maestros eran concebidos como simples "administradores del currículo". En este sentido, el movimiento pedagógico se constituye en la respuesta que estableció el magisterio colombiano a las políticas del Estado; respuesta que se desarrolló a partir de ejercicios de investigación y acción que tenían como fin ubicar a la pedagogía como un saber fundante del hecho educativo y posicionar al maestro como un intelectual orgánico. 
